\title{
A Framework for Obtaining Steady-State Maneuvering Characteristics of Underwater Vehicles Using Sea-Trial Data
}

\author{
$\varnothing y$ yvind Hegrenæs $^{a} \quad$ Oddvar Hallingstad $^{b} \quad$ Bjørn Jalving $^{c}$ \\ ${ }^{a, b}$ Department of Engineering Cybernetics, \\ Norwegian University of Science and Technology, NO-7491 Trondheim, Norway \\ ${ }^{a, b}$ University Graduate Center, NO-2027 Kjeller, Norway \\ ${ }^{c}$ Kongsberg Maritime, NO-3191 Horten, Norway
}

\begin{abstract}
This paper reports the development and experimental evaluation of a simple and intuitive framework for obtaining steady-state maneuvering characteristics of a wide class of underwater vehicles. The possible use of steadystate characteristics includes navigation, fault-detection and vehicle design evaluation. The presented algorithms allow unknown currents to be included in the identification loop. Measured or estimated vehicle position, velocity and attitude are required inputs. The effectiveness of the algorithms is illustrated using experimental data collected by the HUGIN 4500 AUV. The identification results are found to be consistent and in good agreement with a physical interpretation.
\end{abstract}

\section{INTRODUCTION}

The maneuvering characteristics of an ocean vehicle are typical measures of the ability to control the trajectory, speed, and orientation of the vehicle at sea. With the increasing focus on performance, efficiency, and safety, several (yet no definitive) standards have been proposed for identifying such characteristics, e.g. [1], [2]. In accordance to these standards, the appropriate experiments can be carried out using either a full-scale vehicle or by doing model tests. Common model test are free-running tests (self-actuated, resemble full-scale trials) and captive tests (forced movement with controls fixed). For additional information on the various approaches the reader is referred to [1], [3], [4], [5], and references therein.

The purpose of this paper is twofold. First, a general mathematical framework is established for obtaining stationary speed and turning characteristics of underwater vehicles using data from full-scale sea-trials. Since the characteristics can be significantly affected by current, this is explicitly accounted for during identification. Second, the proposed algorithms are evaluated using experimental data collected by the HUGIN 4500 survey AUV (autonomous underwater vehicle). The presented framework is thought to be applicable to a wide class of submersibles.

Besides the obvious application to vehicle design evaluation and validation, a possible use of steady-state maneuvering characteristics includes navigation and faultdetection. In [6] the water-relative speed was estimated from steady-state propeller rotation rate, and then included in the navigation system. Equally, if the current is fully or partially (bounds) known, it can be used together with the characteristics to detect measurement failures or outliers in the real-time navigation system.

This paper is organized as follows. Section II reports a general mathematical framework for obtaining the vehicle steady-state characteristics. Section III describes our experimental setup. The experimental evaluation of the proposed framework is reported in Section IV.

\section{Problem Statement And Identification}

In this section, a mathematical framework is given for identifying the steady-state maneuvering characteristics of an underwater vehicle using experimental field data. We assume the presence of a constant or slowly varying current whose magnitude and direction is unknown. It is furthermore assumed that the current is irrotational and two-dimensional. In the proposed algorithms, the current is identified together with the vehicle related parameters. Measured or estimated vehicle position, velocity and attitude are required inputs.

\section{A. Modeling}

With the assumption that the vehicle operates in a limited geographical area, it is usually sufficient to use a local flat Earth approximation when describing its location. Let $\{m\}$ denote an Earth fixed coordinate frame, where the origin is located at the surface of the WGS84 Earth ellipsoid, and the orientation is north-east-down (NED). Similarly, let $\{w\}$ denote a reference frame where the origin is fixed to, and translates with the water (due to current). Note that $\{w\}$ does not rotate relative to $\{m\}$. The last frame $\{b\}$ is a body fixed reference frame where the axes coincide with the principal axes of the vehicle. The origin of $\{b\}$ is typically placed at the vehicle center of buoyancy or the center of gravity. A general expression of the vehicle position can now be written as

$$
\begin{aligned}
\boldsymbol{p}_{m b}^{m} & =\boldsymbol{p}_{m w}^{m}+\boldsymbol{p}_{w b}^{m} \\
& =\boldsymbol{p}_{m w}^{m}+\boldsymbol{R}_{w}^{m} \boldsymbol{p}_{w b}^{w},
\end{aligned}
$$

where $\boldsymbol{p}_{w b}^{m} \in \mathbb{R}^{3}$ is the position vector from the origin of $\{w\}$ to the origin $\{b\}$, decomposed in $\{m\}$, and $\boldsymbol{R}_{w}^{m} \in$ $S O(3)$ is the rotation matrix from $\{m\}$ to $\{w\}$, or equally, the coordinate transformation matrix from $\{w\}$ to $\{m\}$. The velocity of $\{b\}$ relative to $\{m\}$, represented in $\{m\}$, 


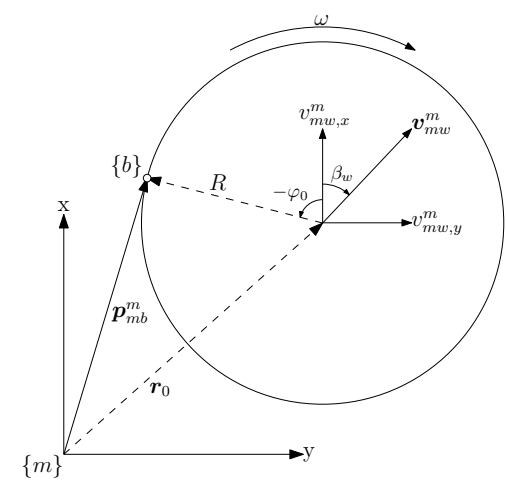

Fig. 1. Horizontal turning maneuver in the presence of a time-invariant current. The position $\boldsymbol{p}_{m b}^{m}$ is time-varying; initial position marked $\circ$.

is given as $\boldsymbol{v}_{m b}^{m}:=\dot{\boldsymbol{p}}_{m b}^{m}$, or decomposed in $\{b\}$ as $\boldsymbol{v}_{m b}^{b}:=$ $\boldsymbol{R}_{m}^{b} \boldsymbol{v}_{m b}^{m}$. The interpretation of the other variables follows directly. Taking the derivative of both sides of (1) yields

$$
\begin{aligned}
\dot{\boldsymbol{p}}_{m b}^{m} & =\dot{\boldsymbol{p}}_{m w}^{m}+\dot{\boldsymbol{R}}_{w}^{m} \boldsymbol{p}_{w b}^{w}+\boldsymbol{R}_{w}^{m} \dot{\boldsymbol{p}}_{w b}^{w} \\
& =\dot{\boldsymbol{p}}_{m w}^{m}+\boldsymbol{R}_{w}^{m} \dot{\boldsymbol{p}}_{w b}^{w},
\end{aligned}
$$

where $\dot{\boldsymbol{R}}_{w}^{m}$ equals zero due to the assumption of an irrotational current. Substituting for the derivatives, and multiplying both sides of (2) with $\boldsymbol{R}_{m}^{b}$ gives the velocity relationship

$$
\boldsymbol{v}_{m b}^{b}=\boldsymbol{R}_{m}^{b} \boldsymbol{v}_{m w}^{m}+\boldsymbol{v}_{w b}^{b} .
$$

A different representation can be derived for the case where the vehicle performs a steady turning maneuver, under the influence of a constant current. From the geometric entities in Fig. 1, it is straightforward to derive the equation

$$
\boldsymbol{p}_{m b}^{m}=\boldsymbol{r}_{0}+\boldsymbol{v}_{m w}^{m} t+\left[\begin{array}{c}
\cos \left(\omega t-\varphi_{0}\right) \\
\sin \left(\omega t-\varphi_{0}\right) \\
0
\end{array}\right] R
$$

where $t$ is a free variable denoting time, and $\boldsymbol{r}_{0}, \varphi_{0}$ are initial conditions at $t=0$. The circle with radius $R$ represents the path that the origin of $\{b\}$ would follow if no current was present, and $\omega$ is the angular rate of the same point about the origin of the circle, which location is given by $\boldsymbol{r}_{0}$. Note that $\omega$ should not be confused with the vehicle heading rate.

The expressions in (3) and (4) are both valid when the entries of $\boldsymbol{v}_{m w}^{m}$ are all non-zero. As noted earlier however, it is assumed throughout this paper that the vertical current component, or equally, the third entry of $\boldsymbol{v}_{m w}^{m}$ is zero at all time. Based on this assertion we can rewrite (3) as

$$
\boldsymbol{v}_{m b}^{b}=\left[\begin{array}{ll}
\boldsymbol{c}_{1} & \boldsymbol{c}_{2}
\end{array}\right]\left[\begin{array}{l}
v_{m w, x}^{m} \\
v_{m w, y}^{m}
\end{array}\right]+\boldsymbol{v}_{w b}^{b}
$$

where $c_{i}$ denotes the $i$-th column of the rotation matrix $\boldsymbol{R}_{m}^{b}$. The subsystem of interest in (4) is restated as

$$
\left[\begin{array}{l}
p_{m b, x}^{m} \\
p_{m b, y}^{m}
\end{array}\right]=\left[\begin{array}{l}
r_{0, x} \\
r_{0, y}
\end{array}\right]+\left[\begin{array}{l}
v_{m w, x}^{m} \\
v_{m w, y}^{m}
\end{array}\right] t+\left[\begin{array}{c}
\cos \left(\omega t-\varphi_{0}\right) \\
\sin \left(\omega t-\varphi_{0}\right)
\end{array}\right] R
$$

While (5) allows for a varying current, we will for the remainder assume that the current is piecewise constant on each subset of data used during identification. In general, the current is assumed to be constant or slowly varying.

\section{B. Least-Squares Identification}

The deterministic least-squares (LS) scheme is a well known and widely used approach for fitting data and for identifying model parameters in both linear and nonlinear overdetermined systems. For a thorough discussion on the topic, the reader is referred to the numerous textbooks available, e.g. [7]. As used herein, LS can be applied to the models in (5) and (6) in order to obtain steady-state maneuvering characteristics of an underwater vehicle. Also, since the unknown current is included in the models it is identified simultaneously.

By defining $\boldsymbol{z}:=\boldsymbol{v}_{m b}^{b}$, we have from (5) that

$$
\underbrace{\left[\begin{array}{c}
\boldsymbol{z}\left(t_{1}\right) \\
\boldsymbol{z}\left(t_{2}\right) \\
\vdots \\
\boldsymbol{z}\left(t_{N}\right)
\end{array}\right]}_{\boldsymbol{Z} \in \mathbb{R}^{3 N \times 1}}=\underbrace{\left[\begin{array}{ccc}
\boldsymbol{I}_{3 \times 3} & \boldsymbol{c}_{1}\left(t_{1}\right) & \boldsymbol{c}_{2}\left(t_{1}\right) \\
\boldsymbol{I}_{3 \times 3} & \boldsymbol{c}_{1}\left(t_{2}\right) & \boldsymbol{c}_{2}\left(t_{2}\right) \\
\vdots & \vdots & \vdots \\
\boldsymbol{I}_{3 \times 3} & \boldsymbol{c}_{1}\left(t_{N}\right) & \boldsymbol{c}_{2}\left(t_{N}\right)
\end{array}\right]}_{\boldsymbol{H} \in \mathbb{R}^{3 N \times 5}} \boldsymbol{\theta},
$$

where $\boldsymbol{\theta}:=\operatorname{col}\left(\boldsymbol{v}_{w b}^{b}, v_{m w, x}^{m}, v_{m w, y}^{m}\right) \in \mathbb{R}^{5}$ is a constant column vector of the unknown model parameters, $N$ is the number of samples, and $t_{1}, t_{2}, \ldots, t_{N}$ represent the sample times. If $\boldsymbol{Z}$ and $\boldsymbol{H}$ are fully known or measured, then it is possible to obtain an LS estimate of $\boldsymbol{\theta}$ by solving the optimization problem

$$
\arg \min _{\boldsymbol{\theta}}[\boldsymbol{Z}-\boldsymbol{H} \boldsymbol{\theta}]^{\top}[\boldsymbol{Z}-\boldsymbol{H} \boldsymbol{\theta}] .
$$

In order for a solution to exist, $\boldsymbol{\theta}$ must be observable. A large condition number of $\boldsymbol{H}$ indicates marginal (poor) observability [8]. Analogous, a condition number close to unity indicate that the problem is numerically well-posed.

Now let $\boldsymbol{\Phi}:=\operatorname{col}\left(r_{0, x}, r_{0, y}, v_{m w, x}^{m}, v_{m w, y}^{m}, \omega, \varphi_{0}, R\right) \in$ $\mathbb{R}^{7}$ be a vector of constant parameters, and from (6) define

$$
\boldsymbol{h}\left(\boldsymbol{\Phi}, t_{i}\right):=\left[\begin{array}{l}
r_{0, x} \\
r_{0, y}
\end{array}\right]+\left[\begin{array}{l}
v_{m w, x}^{m} \\
v_{m w, y}^{m}
\end{array}\right] t_{i}+\left[\begin{array}{c}
\cos \left(\omega t_{i}-\varphi_{0}\right) \\
\sin \left(\omega t_{i}-\varphi_{0}\right)
\end{array}\right] R
$$

Under the assumption that $\boldsymbol{y}:=\operatorname{col}\left(p_{m b, x}^{m}, p_{m b, y}^{m}\right) \in \mathbb{R}^{2}$ is fully known or measured, it is possible to obtain an LS estimate of $\boldsymbol{\Phi}$ by solving the optimization problem

$$
\arg \min _{\mathbf{\Phi}} \sum_{i=1}^{M}\left[\boldsymbol{y}\left(t_{i}\right)-\boldsymbol{h}\left(\boldsymbol{\Phi}, t_{i}\right)\right]^{\top}\left[\boldsymbol{y}\left(t_{i}\right)-\boldsymbol{h}\left(\boldsymbol{\Phi}, t_{i}\right)\right],
$$

where $M$ and $t_{i}$ denote the number of samples and sample times, respectively. Again, observability conditions apply.

Many approaches exist for solving the linear and nonlinear LS problems formulated above. Not surprisingly, most results have been published for the linear case in (8). Care must be taken when solving the nonlinear problem in (10), due to the sensitivity to initial conditions and the possibility of local minima. Several algorithms are available in MATLAB, some of which will be utilized in this paper. For a comprehensive treatment on numerical LS methods, the reader is referred to [9].

In some cases the measured data may vary in quality. To model this situation it is possible to include weights in the LS problem formulation. Typically this is done by incorporating the measurement error covariances. Weights are not included in this paper. 


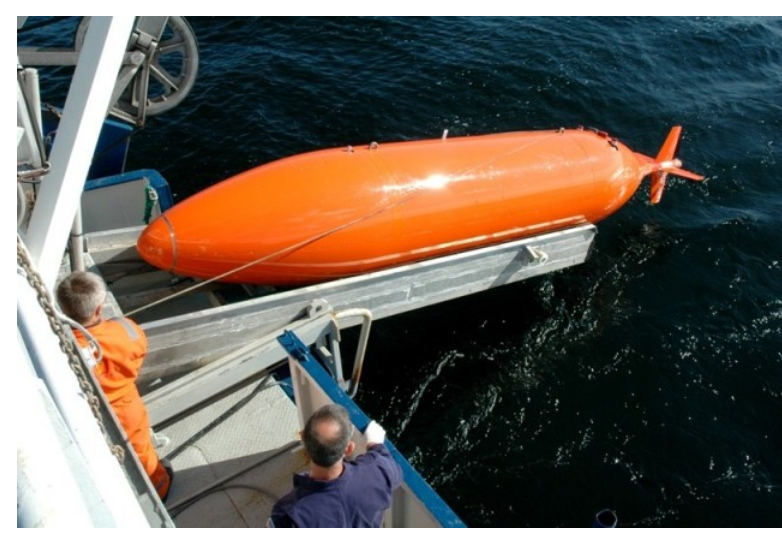

Fig. 2. The HUGIN 4500 AUV during sea-trial launch.

\section{EXPERIMENTAL SETUP}

Navigation data collected by the HUGIN 4500 AUV are used as reference and basis throughout this paper. An overview of vehicle particulars is given subsequently, followed by a description of the experiments conducted in order to evaluate the methods outlined in Section II.

\section{A. Vehicle Specifications}

The Kongsberg Maritime manufactured HUGIN 4500 is the latest member of the HUGIN AUV family. Figure 2 shows a picture from one of the trials in September 2006.

The overall length of the vehicle is approximately 6.5 $\mathrm{m}$ and the maximum diameter is $1 \mathrm{~m}$, yielding a total displacement of nearly $3.4 \mathrm{~m}^{3}$. The nominal dry mass is $1950 \mathrm{~kg}$ and the vehicle is close to neutrally buoyant. Designed using titanium, glass, carbon fiber laminate, and high performance syntactic foam, HUGIN 4500 can operate at depths down to $4500 \mathrm{~m}$.

Power is provided by an aluminum/oxygen semi-fuel cell battery system, sufficient for up to 60-70 hours endurance, depending on the payload configuration and propulsion. The nominal cruising speed of the vehicle is about 3.7 knots or $1.9 \mathrm{~m} / \mathrm{s}$.

For propulsion HUGIN 4500 is fitted with a high torque synchronous motor, allowing direct axial drive of a single large three-bladed propeller. A cruciform tail configuration with four identical control surfaces is used for maneuvering. The vehicle is passively stable in roll and no effort is currently done for controlling this degree of freedom. As for high-level guidance, HUGIN 4500 can operate in either UUV (unmanned underwater vehicle) or AUV mode. In AUV mode the vehicle operates without supervision, and independently of the mother ship. After being launched it autonomously executes the survey as specified in a predetermined mission plan, before returning to a desired pick-up location. In UUV mode the vehicle is maneuvered close to the mother ship, hence enabling real-time supervision, operator commands and mission plan changes via an acoustic data link. In both operation modes several control strategies can be chosen, including waypoint or heading mode, and depth or altitude (terrain following capability) mode. The experimental
TABLE I

IMU SPECIFICATIONS

\begin{tabular}{c|c|c|c}
\hline Model & Gyro Technology & Gyro Bias & Accelerometer Bias \\
\hline IXSEA IMU90 & Fiber optic & $\pm 0.05^{\circ} / \mathrm{h}$ & $\pm 500 \mu \mathrm{g}$ \\
\hline
\end{tabular}

TABLE II

PRIMARY NAVIGATION AIDING SENSORS

\begin{tabular}{c|l|c|c}
\hline Variable & Sensor & Precision & Rate \\
\hline Position & Kongsberg HiPAP & Range, Angle: $<20 \mathrm{~cm}, 0.12^{\circ}$ & Varying* \\
Velocity & RDI WHN 300 & $\pm 0.4 \% \pm 0.2 \mathrm{~cm} / \mathrm{s}$ & $1 \mathrm{~Hz}$ \\
Pressure & Paroscientific & $0.01 \%$ full scale & $1 \mathrm{~Hz}$ \\
\hline \multicolumn{2}{c}{ * Data rate is approximately $1 / 3 \mathrm{~Hz}$. In real-time HUGIN receives position }
\end{tabular}

updates at about $1 / 30 \mathrm{~Hz}$, from the surface vessel via an acoustic link.

data analyzed in this paper were collected while operating in UUV mode, and with depth and waypoint control.

HUGIN 4500 is equipped with an inertial navigation system (INS) which calculates position, velocity and attitude using high frequency data from an inertial measurement unit (IMU). Some of the IMU specifications are listed in Table I. In order to obtain a low drift navigation solution, a Kalman filter is implemented which utilizes a variety of navigation sensors for aiding the INS. In UUV mode the surface ship tracks the submersible with an ultra short baseline acoustic position system (USBL). By combining DGPS with USBL, a global position estimate of the AUV can be obtained. This estimate is then sent to the AUV on the acoustic communication link. Additional navigation sensors include compass, pressure sensor, and Doppler velocity log (DVL). Primary aiding sensors and some of their specifications are listed in Table II. Readers are referred to [10], [11] for additional information on the navigation system and navigation accuracy.

\section{B. Mission Description}

During September and October 2006, several sea-trials were conducted with the HUGIN 4500 AUV in the vicinity of $59^{\circ} 29^{\prime} \mathrm{N}, 10^{\circ} 28^{\prime} \mathrm{E}$, in the Oslo-fjord close to Horten. All together, more than 60 hours of data were collected, in which a subset of approximately 6 hours is utilized in this paper. The test area is shown in Fig. 3, where the resolution of the map is about $10 \mathrm{~m}$.

In light of Section II, data were gathered with the purpose of obtaining steady-state maneuvering characteristics for both straight-line motion and turning maneuvers. In the first part, the vehicle was kept at a constant depth while moving along square-shaped trajectories. The propeller rotation rate was held fixed throughout the completion of a full square. The sides of the individual squares ranged from 450-600 $\mathrm{m}$ in length, sufficient for the vehicle to retain steady-state velocity between 90 degree turns or after changing the propeller rotation rate. It took the vehicle about 20 minutes to complete one square. During this time slot it was assumed that the current was constant and two-dimensional. The choice of square-shaped trajectories was decided in order to enhance observability. The condition numbers for $\boldsymbol{H}$ in (8) were all found to be close to unity. An example of data gathered during these experiments can be seen in Fig. 4, with the corresponding trajectory shown in Fig. 3. 


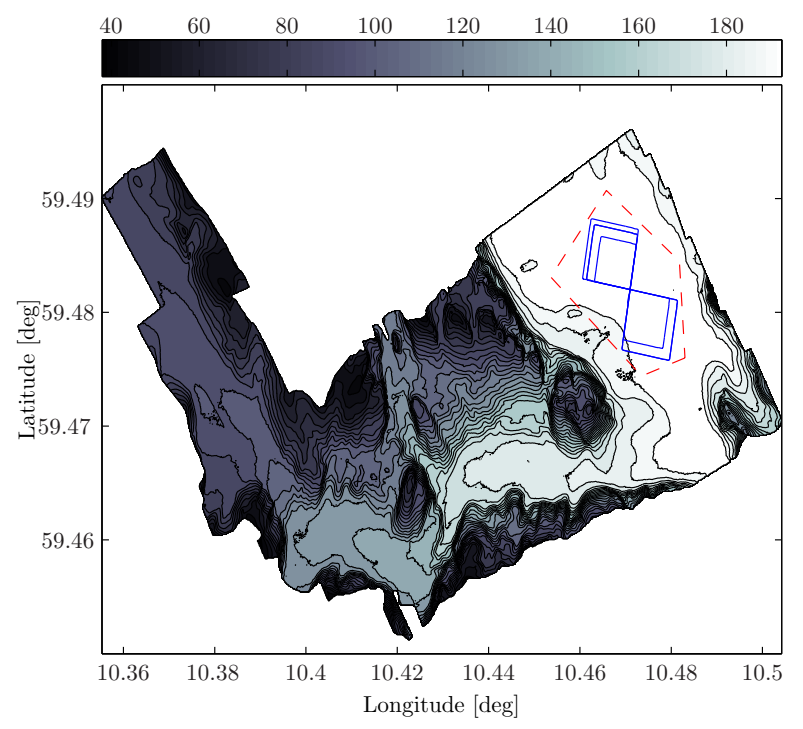

Fig. 3. Test area. All the experiments were conducted within the dashed area. The solid trajectory corresponds to the data in Fig. 4.

As for the experiments involving turning maneuvers, these were also conducted while operating at constant depths. Unlike in the square-shaped experiments, additional operator commands were also transmitted to the vehicle in real-time. Specifically, this included fixed setpoints for the propeller rotation rate and for the rudders. After deflecting the rudders, they were kept in locked position while applying a selection of different propeller rotation rates. The various set-points remained unchanged up to when steady-state turning conditions were obtained. Each iteration took less than 8 minutes to complete. As before, the current was assumed to be two-dimensional and constant during the execution of each iteration. Both left and right turn maneuvers were done. An example of collected data is seen in Fig. 5.

\section{EXPERIMENTAL RESUlTS}

This section reports the experimental evaluation of the proposed mathematical framework. Since experimental sea-trial data typically contain unknown disturbances due to current, this effect must be identified and properly accounted for. In the following, various results are presented, succeeded by a discussion on the validity of the identified parameters, including the current.

Prior to applying the proposed algorithms, the data were wild-point filtered and smoothed in order to enhance accuracy. This was done using the post-processing tool NavLab [12]. The smoothed vehicle position, velocity and attitude were then utilized during identification, after doing appropriate data extraction. The latter was done in two parts, where the first part involved extracting the data corresponding to one complete square or turning maneuver. Second, the transient parts of the extracted data were discarded. The identification was finally completed for each extracted subset of data, one at a time.

\section{A. Identification Results}

A summary of the LS identification results is given in Table III and IV, for the turning and square maneuvers
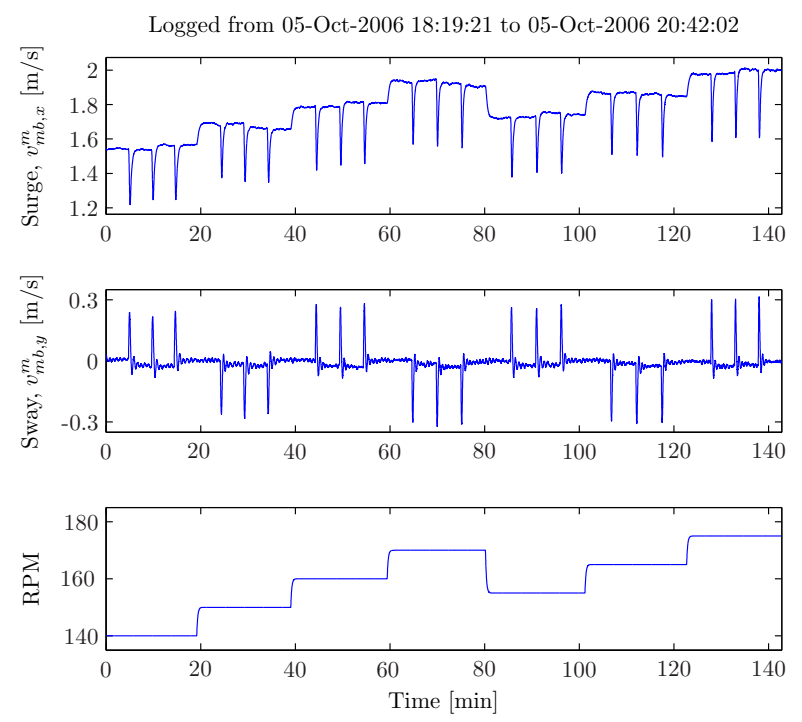

Fig. 4. Example of square maneuvers with 7 successive squares. The spikes clearly show the coupling between surge and sway.
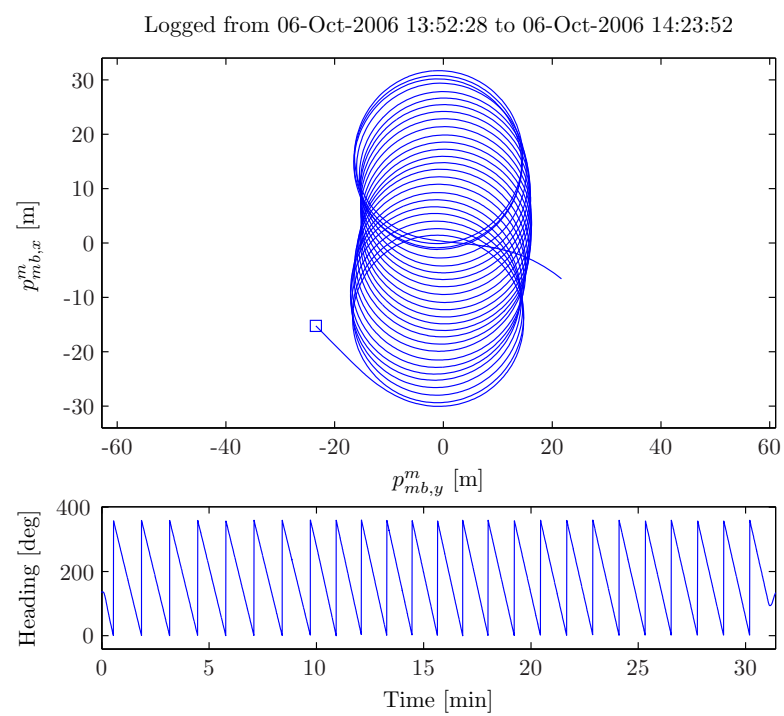

Fig. 5. Turning maneuvers. The rudders were kept in locked position while varying the RPM. The current induced drift is clearly observed.

respectively. For the estimated current, the magnitude $\left\|\boldsymbol{v}_{m w}^{m}\right\|$ is taken as the Euclidian norm of $\boldsymbol{v}_{m w}^{m}$, where the third entry of $\boldsymbol{v}_{m w}^{m}$ equals zero by assumption. The current direction $\beta_{w}$ is calculated from

$$
\beta_{w}=\operatorname{atan} 2\left(v_{m w, y}^{m}, v_{m w, x}^{m}\right),
$$

where $\beta_{w} \in[0,360)$ is relative North with positive rotation clockwise, i.e. $\beta_{w}=90$ [deg] yields East direction.

As for the results associated with the square maneuvers, the main parameter of interest is the velocity of the vehicle relative to the surrounding fluid. Figure 6 shows the identified $\mathrm{x}$-components (surge), together with a curvefitted function relating the same values to the propeller rotation rate. Note that only a subset of the available data was used in the fitting process, while all the data were used for validation. The mean and standard deviation of the complete set of residuals are 3.3 and $5.9 \mathrm{~mm} / \mathrm{s}$, respectively. The identified y-components (sway) are shown in Fig. 7, together with the estimated vehicle 

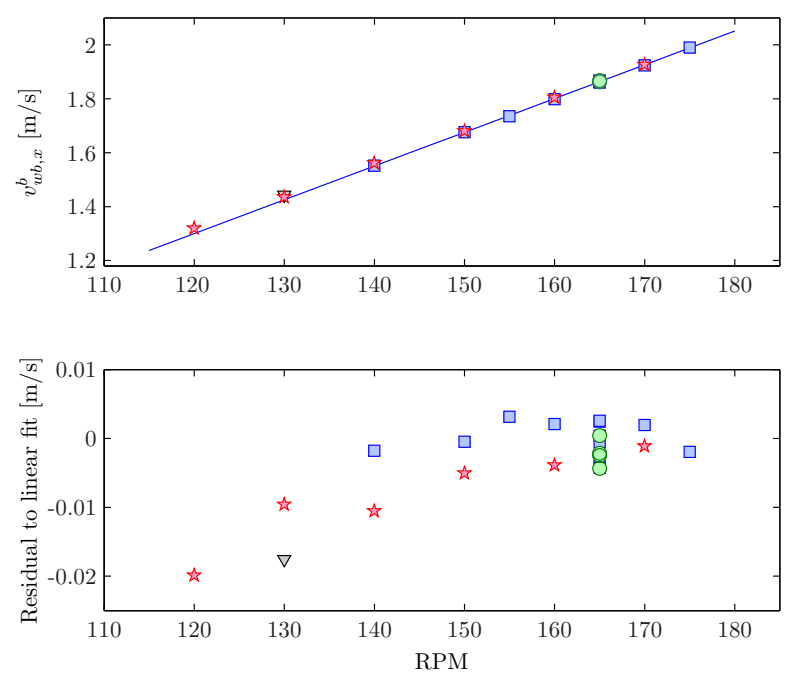

Fig. 6. Square maneuver identification. Solid line: linear fit to the data from Oct 5 and $6(\square)$. Other data: Sep $28(0)$, Sep 26 (出), Sep $7(\nabla)$.
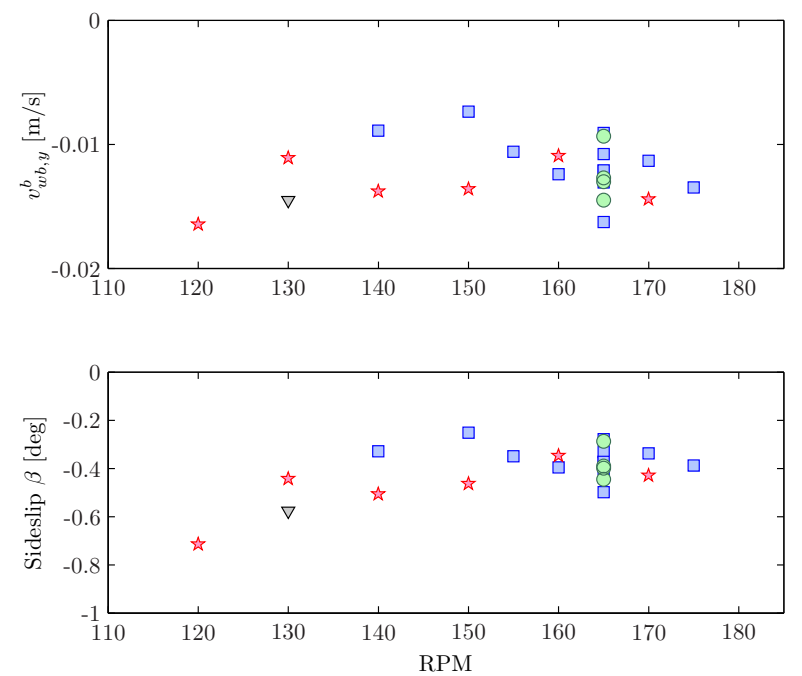

Fig. 7. Square maneuver identification. Similar labels as in Fig. 6.

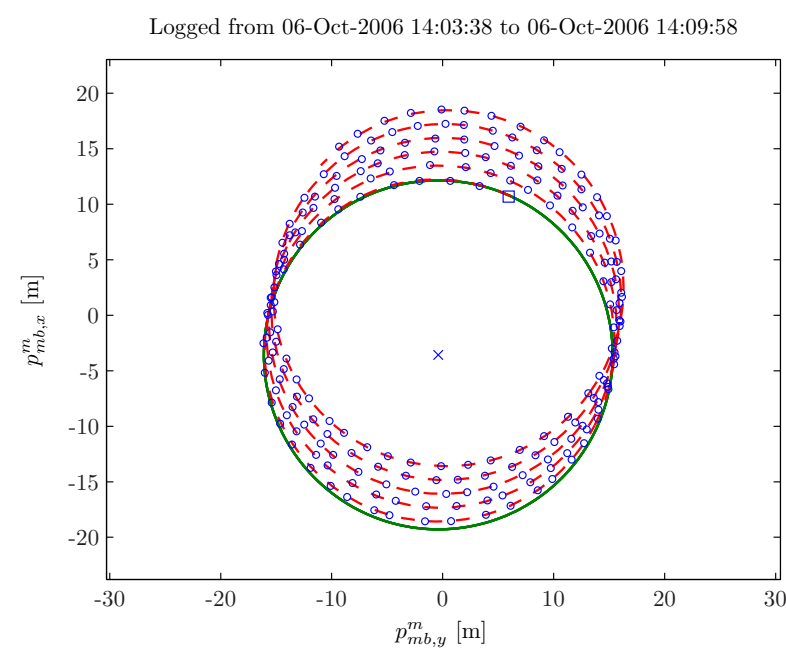

Fig. 8. Turn maneuver identification. Solid circle: path that the vehicle would follow without any current present. Other labels: circle origin $(\times)$, initial position $(\square)$, measured position (O), LS fitted path (dashed).
TABLE III

IDENTIFICATION RESULTS FOR TURNING MANEUVERS*

\begin{tabular}{c|c|c|c|c|c|r}
\hline Time & RPM & $\delta$ & $R$ & $\omega$ & $\left\|\boldsymbol{v}_{m w}^{m}\right\|$ & \multicolumn{1}{c}{$\beta_{w}$} \\
\hline Sep 20 @ 13:50-13:57 & 155 & -12.75 & +15.6 & +4.8 & 0.009 & 107.8 \\
Sep 26 @ 15:06-15:10 & 155 & -12.76 & +15.4 & +4.8 & 0.010 & 208.9 \\
Sep 26 @ 15:11-15:16 & 165 & -12.76 & +15.6 & +5.2 & 0.013 & 191.8 \\
Sep 26 @ 15:18-15:24 & 175 & -12.75 & +15.8 & +5.5 & 0.008 & 182.6 \\
Oct 06 13:54-14:02 & 150 & +12.22 & +15.7 & -4.6 & 0.017 & 0.5 \\
Oct 06 14:03-14:10 & 165 & +12.21 & +15.7 & -5.1 & 0.018 & 9.2 \\
Oct 06 @ 14:12-14:20 & 160 & +12.22 & +15.7 & -4.9 & 0.018 & 356.1 \\
Oct 12 @ 13:53-13:58 & 150 & -12.76 & +16.1 & +4.5 & 0.014 & 173.2 \\
Oct 12 @ 14:00-14:05 & 165 & -12.77 & +16.2 & +5.0 & 0.009 & 197.9 \\
Oct 12@ 14:07-14:12 & 175 & -12.75 & +16.2 & +5.4 & 0.007 & 197.4 \\
\hline
\end{tabular}

TABLE IV

IDENTIFICATION RESULTS FOR SQUARE MANEUVERS*

\begin{tabular}{l|c|c|c|c|c|r}
\hline Time & RPM & $v_{w b, x}^{b}$ & $v_{w b, y}^{b}$ & $v_{w b, z}^{b}$ & $\left\|\boldsymbol{v}_{m w}^{m}\right\|$ & \multicolumn{1}{|c}{$\beta_{w}$} \\
\hline Sep 07 @ 12:57-13:10 & 130 & 1.443 & -0.015 & -0.082 & 0.006 & 169.1 \\
Sep 26 @ 13:04-13:22 & 120 & 1.320 & -0.016 & -0.109 & 0.002 & 30.3 \\
Sep 26 @ 13:31-13:43 & 130 & 1.435 & -0.011 & -0.099 & 0.003 & 30.5 \\
Sep 26 @ 13:45-14:02 & 140 & 1.561 & -0.013 & -0.090 & 0.004 & 123.6 \\
Sep 26 @ 14:05-14:22 & 150 & 1.681 & -0.013 & -0.085 & 0.004 & 223.0 \\
Sep 26 @ 14:25-14:42 & 160 & 1.805 & -0.011 & -0.077 & 0.007 & 172.0 \\
Sep 26 @ 14:44-14:57 & 170 & 1.927 & -0.014 & -0.072 & 0.010 & 115.8 \\
Sep 28 @ 14:35-14:47 & 165 & 1.865 & -0.013 & -0.015 & 0.002 & 17.1 \\
Sep 28 @ 14:49-15:04 & 165 & 1.868 & -0.013 & -0.015 & 0.005 & 256.5 \\
Sep 28 @ 15:07-15:20 & 165 & 1.863 & -0.010 & -0.015 & 0.003 & 358.4 \\
Sep 28 @ 15:22-15:36 & 165 & 1.866 & -0.014 & -0.015 & 0.005 & 275.5 \\
Oct 05 @ 18:20-18:39 & 140 & 1.552 & -0.009 & +0.074 & 0.018 & 138.0 \\
Oct 05 @ 18:41-18:58 & 150 & 1.676 & -0.008 & +0.067 & 0.019 & 149.7 \\
Oct 05 @ 19:01-19:19 & 160 & 1.799 & -0.012 & +0.062 & 0.020 & 144.6 \\
Oct 05 @ 19:22-19:39 & 170 & 1.924 & -0.012 & +0.057 & 0.018 & 177.3 \\
Oct 05 @ 19:41-20:00 & 155 & 1.735 & -0.011 & +0.063 & 0.017 & 156.7 \\
Oct 05 @ 20:04-20:22 & 165 & 1.861 & -0.011 & +0.057 & 0.012 & 179.5 \\
Oct 05 @ 20:24-20:42 & 175 & 1.990 & -0.013 & +0.051 & 0.015 & 159.4 \\
Oct 06 @ 00:47-01:02 & 165 & 1.867 & -0.009 & +0.051 & 0.022 & 338.1 \\
Oct 06 @ 02:06-02:20 & 165 & 1.864 & -0.013 & +0.049 & 0.015 & 17.2 \\
Oct 06 @ 03:01-03:21 & 165 & 1.861 & -0.012 & +0.049 & 0.018 & 305.5 \\
Oct 06 @ 04:15-04:34 & 165 & 1.863 & -0.012 & +0.048 & 0.004 & 301.7 \\
Oct 06 @ 07:31-07:46 & 165 & 1.867 & -0.016 & +0.042 & 0.021 & 162.7 \\
\hline * Units in order: [rev $/ \mathrm{min}, \mathrm{m} / \mathrm{s}, \mathrm{m} / \mathrm{s}, \mathrm{m} / \mathrm{s}, \mathrm{m} / \mathrm{s}, \mathrm{deg}]$ &
\end{tabular}

sideslip. The mean and standard deviation are -0.0122 $\mathrm{m} / \mathrm{s}$ and $2.1 \mathrm{~mm} / \mathrm{s}$ for the sway components, and -0.4 and 0.09 degree for the sideslip. The standard deviations indicate an insignificant correlation with the propeller rotation rate. The identification results for one of the turning maneuvers are visualized in Fig. 8. The LS fitted trajectory matches the measured data well. Similar results were also obtained for the other turning maneuvers. The standard deviations of the fitting errors were in the order of $0.15 \mathrm{~m}$ or lower. While turning radius and turning rate are the main parameters of interest, it is straightforward to obtain an estimate of the water-relative velocity during turning maneuvers, once the current has been estimated. This can be done using the expression in (5).

\section{B. Discussion}

Are the identified parameters reasonable? Are the estimated currents consistent with a physical understanding?

The total current is made up from tidal current and wind-generated current. The latter contribution is assumed negligible since all the experiments in this paper were conducted at depths of 125-160 m. Tidal currents are the horizontal currents associated with the vertical rise and fall of the tide. The current resulting from a rising tide is called flood, and equally ebb for a falling tide. Slack water occurs during the time interval between the two, when the 

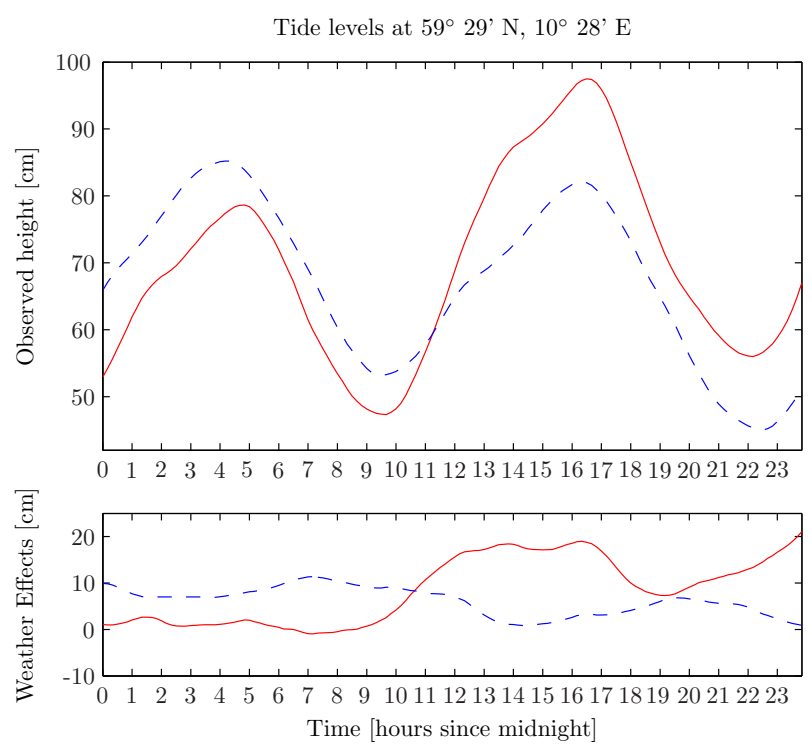

Fig. 9. Tide levels at test site. Dashed and solid lines: Oct 5 and 6, respectively. Observed height is relative Chart datum. Courtesy to [13].

water has no horizontal motion. In fjords like the Oslofjord, the current can only run in two opposite directions, with possible local variations due to topography [13].

From its southern inlet by the Skagerak sea, the Oslofjord stretches straight north. In light of Fig. 3 and the discussion above, it seems reasonable that the flood will be directed to the north or slightly north-west and the ebb to the south or slightly south-east. This is in good agreement with the identified current directions. The tide levels at the test site for Oct 5 and 6 are shown in Fig. 9. The data from Oct 26 were collected during and close to slack water conditions. An examination of the current speeds obtained from the square and turning maneuvers reveals that the two (very different) LS formulations yield results of the same order of magnitude. The lowest amplitudes were found to occur during or close to slack water conditions, as anticipated.

The identified steady-state turning characteristics summarized in Table III are consistent, and they seem reasonable. As expected, an increase in propeller RPM yields a slightly larger turning radius and turning rate.

The steady-state straight-line motion parameters in Table IV also appear plausible. The identification results for the different data-sets show only small variations in the surge and sway components. For the 10 independent data-sets logged at 165 RPM, the standard deviations for surge and sway are $2.5 \mathrm{~mm} / \mathrm{s}$ and $2.1 \mathrm{~mm} / \mathrm{s}$, respectively. The identified velocity $\mathrm{z}$-components (heave) are less conclusive, yet still as expected. The major differences are attributed to slightly different ballasting, hence to the nominal pitch of the vehicle. Other variations are due to an increase or decrease in the propeller RPM (pitch and heave level out as the RPM is increased), and the slowly change of ballasting with time (the HUGIN 4500 battery decreases its mass with $0.5 \mathrm{~kg} /$ hour). Note that the other maneuvering parameters are, by far, less sensitive to ballasting and pitch offsets than the heave components.

\section{CONCLUSIONS AND FURTHER WORK}

A mathematical framework has been proposed for obtaining steady-state maneuvering characteristics of a wide class of underwater vehicles. The presented algorithms allow unknown currents to be included in the identification loop. Vehicle position, velocity and attitude are required inputs. The effectiveness of the algorithms has been shown using experimental data collected by the HUGIN 4500 survey AUV. The identified parameters are in good agreement with a physical interpretation.

\section{A. Further Work}

In order to fully evaluate the proposed algorithms, additional current measurements should be obtained using a current profiler or possibly the DVL. At the time the experiments were conducted this was not available.

Due to time constraints, only a small selection of rudder deflections was used during the turning maneuvers. In order to establish a functional relationship between rudder deflections, propeller RPM, and steady-state turning characteristics, additional data should be obtained.

\section{ACKNOWLEDGMENT}

The first author acknowledges the financial support from the Research Council of Norway, Kongsberg Maritime, and Kongsberg Defence \& Aerospace, through the UNaMap program. Thanks also to the HUGIN staff and the crew of M/S Simrad Echo for all their help.

\section{REFERENCES}

[1] International Towing Tank Conference (ITTC). [Online]. Available: http://ittc.sname.org/documents.htm

[2] International Maritime Organization (IMO).

[3] C. L. Crane, H. Eda, and A. Landsburg, "Motions in waves and controllability," in Principles of Naval Architecture, E. V. Lewis, Ed. Jersey City, NJ: Society of Naval Architects and Marine Engineers, 1988, vol. 3, ISBN 0-939773-02-3.

[4] J. P. Feldman, "Method of performing captive-model experiments to predict the stability and control characteristics of submarines," Naval Surface Warfare Center, Maryland, Tech. Rep. CRDKNSWC-HD-0393-25, June 1995.

[5] J. Journée and J. Pinkster, "Introduction in ship hydromechanics," 2002, Lecture notes. Last acceced on January 20 2007. [Online]. Available: http://www.ocp.tudelft.nl/mt/journee/

[6] A. Gadre and D. Stilwell, "A complete solution to underwater navigation in the presence of unknown currents based on range measurements from a single location," in Intelligent Robots and Systems, 2005. (IROS 2005). 2005 IEEE/RSJ International Conference on, 2005, pp. 1420-1425.

[7] L. Ljung, System Identification: Theory for the User, 2nd ed. Prentice Hall PTR, 1999, ISBN 0-13-656695-2.

[8] Y. Bar-Shalom, X.-R. Li, and T. Kirubarajan, Estimation with Applications to Tracking and Navigation. Wiley, 2001, ISBN 0-471-41655-x.

[9] A. Björck, Numerical methods for least squares problems. Philadelphia: SIAM, 1996, ISBN 0-89871-360-9.

[10] B. Jalving, K. Gade, O. Hagen, and K. Vestgård, "A toolbox of aiding techniques for the HUGIN AUV integrated inertial navigation system," in OCEANS 2003, Proceedings, vol. 2, San Diego, CA, USA, 2003, pp. 1146-1153.

[11] B. Jalving, K. Vestgård, and N. Størkersen, "Detailed seabed surveys with AUVs," in Technology and Applications of Autonomous Underwater Vehicles, G. Griffiths, Ed. London: Taylor \& Francis, 2003, vol. 2, pp. 179-201, ISBN 0-415-30154-8.

[12] K. Gade, "NavLab, a generic simulation and post-processing tool for navigation," European Journal of Navigation, vol. 2, no. 4, pp. 21-59, Nov. 2004.

[13] Norwegian Hydrographic Service web site. [Online]. Available: http://vannstand.statkart.no/Engelsk/strom.php?var=side12 